\title{
Gross anatomical and histological orientation of the lateral intermuscular septum of the upper arm: a descriptive study
}

\author{
Valentin C. Dones III ${ }^{1}$, Ian P. Aguinaldo ${ }^{2}$, Trixie Mycah M. Pulido ${ }^{2}$, Limuel M. Goite ${ }^{2}$, \\ Madelyn A. Gabrieles ${ }^{2}$, Martin E. Estrella ${ }^{2}$, Clarissa D. Tan ${ }^{2}$, John Alfred D. Gargalicana ${ }^{2}$, \\ Andrea Denise C. Limjoco ${ }^{2}$, Ramon Joseph A. Sacdalan ${ }^{2}$ \\ ${ }^{1}$ Center for Health Research on Movement Sciences, College of Rebabilitation Sciences, University of Santo Tomas, Manila, Philippines \\ ${ }^{2}$ Department of Physical Therapy, College of Rehabilitation Sciences, University of Santo Tomas, Manila, Philippines
}

\begin{abstract}
Objectives: The lateral intermuscular septum (LIS) is dense and opaque in gross cadaver dissection. It was suggested as source of pain in elbows with lateral epicondylalgia (LE). The study aimed to measure the length of LIS and to describe the fascia orientation and density of LIS in upper extremities of human cadavers.

Methods: 19 upper extremities of 10 formalin preserved human cadavers were dissected using the fascia sparing approach. The LIS was measured from the level of the lateral epicondyle up to the upper arm. The LIS was harvested $5 \mathrm{~cm}$ above and below the lateral epicondyle and submitted to a laboratory for histological staining. Prepared slides containing stained LIS were observed under high power microscope.

Results: In 17 out of the 19 upper extremities, the LIS attached from the brachioradialis to middle deltoid with a mean of $14.5(13.8-15.1) \mathrm{cm}$. 68 out of the 72 fascia images of LIS were linearly oriented in relation to the lateral epicondyle. The LIS was dense on its distal (16/18) and loose (12/16) on its medial sides.

Conclusion: The fibers of LIS were linearly arranged near the lateral epicondyle in the upper arm connecting the brachioradialis and the deltoid. The LIS was dense and loose on its distal and medial sides, respectively. Considering the arrangement, connection and density of LIS in the upper arm, the LIS contributes to the inherent tightness of the elbow that may be important in handgrip activities. The connection between brachioradialis and middle deltoid by LIS is important in considering the changes in shoulder movement associated with lateral elbow pain in LE.
\end{abstract}

Keywords: brachioradialis muscle; lateral intermuscular septum; upper arm

Anatomy 2015;9(2):66-71 C2015 Turkish Society of Anatomy and Clinical Anatomy (TSACA)

\section{Introduction}

Lateral epicondylalgia (LE) is a clinical condition manifesting as lateral elbow pain commonly provoked by handgrip. ${ }^{[1]}$ Lateral epicondylalgia is prevalent in $1.1-1.3 \%$ in men and $1.1-4 \%$ in women in the general population. ${ }^{[2]}$ Standard provocation tests used in diagnosing LE are Cozen, Mill and Maudsley tests. Resisted wrist extension (Cozen test), quick stretch of forearm extensor muscles (Mill test) and resisted middle finger extension (Maudsley) stress the lateral epicondyle through the forearm extensor muscles replicating lateral elbow pain in in $\mathrm{LE}^{[3-5]}$
In addition to the forearm extensor muscles, the lateral epicondyle, radial nerve and deep fascia were amongst potential sources of lateral elbow pain in LE. ${ }^{[4,6-12]}$ Of these multiple sources of LE, the deep fascia in the upper arm, specifically the lateral intermuscular septum (LIS), was suggested to cause lateral elbow pain in LE. ${ }^{[12,13]}$ The LIS may impinge the traversing radial nerve consequently causing pain on the skin overlying the lateral epicondyle. ${ }^{[13]}$

The LIS has high density of collagen appearing as dense and opaque in gross cadaver dissection. To date, 
scanty literature has described the orientation and density of LIS in the upper arm. The LIS bridges the forearm extensor muscles with the middle deltoid, with no reports on linear measurements of LIS. Considering the density of the LIS in the upper arm, the lateral aspect of the elbow appears to be tight. Pathological tightness in the LIS in the lateral aspect of the elbow may further increase the tension in the lateral aspect of the elbow. ${ }^{[14]}$

Considering that orientation, density and linear measurements of LIS were under-investigated in the current literature, this basic anatomy cadaver study aimed to determine the length of LIS and to describe the fascia orientation and density of LIS.

\section{Materials and Methods}

This study was approved by the University of Santo Tomas - College of Rehabilitation Sciences' Ethics Review Board (Protocol No.: FI 2014-005-OR). 20 upper extremities of 10 middle-aged Filipino formalin-preserved cadavers assigned to the University of Santo Tomas - College of Rehabilitation Sciences were dissected in the Anatomy Laboratory of University of Santo Tomas - Faculty of Medicine and Surgery. Of the 10 cadavers, 8 were males and 2 were females. 19 upper extremities of 10 formalinpreserved cadavers were dissected. One upper extremity was not dissected due to advanced decomposition of tissues. No blotches, lacerations, and bruises were noted on the skin of the dissected upper extremities. The LIS from the dissected upper extremities were histologically processed and evaluated at Microlab, a private institution with 52 years of service in histological investigation of tissues.

The upper extremities of cadavers were dissected using the fascia sparing approach. The following anatomical layers were successively dissected: 1 . skin, 2 . superficial fascia containing fat deposits, and 3. deep fascia. Using the blunt end of the scalpel, the layers were separated from each other. The deep fascia on the lateral aspect of the upper arm was exposed up to the level of the greater tuberosity of the humerus and photographed using a digital camera (Nikon D3000) with clear dot-resolution of 3.0-inch 230,000. Using a Vernier caliper accurate up to $0.05 \mathrm{~mm}$, the length of the deep fascia in the lateral aspect of the upper arm was measured thrice by the physiotherapist.

The deep fascia on the lateral aspect of the upper arm was cut $5 \mathrm{~cm}$ above the lateral epicondyle, $5 \mathrm{~cm}$ below the lateral epicondyle and extended up to the anterior aspect of the upper arm.

The specimen was processed using a rigid Microlab processing protocol (see Appendix A). To keep the orientation of the specimen during Microlab processing, the edges of the specimen were shaped by the medical laboratory technician. The affixed specimen was mounted on glass slides. The fascial orientation of the affixed specimen was observed under high power objectives microscope. To capture the images under the microscope, a camera with resolution of 8 megapixels with 1.5 $\mu$ pixels was used.

To date, there are no standard criteria in characterizing the orientation and density of the fascia on microscope images. Using the microscope images, the orientation of the fascia was described as not linear (graded as ' 1 ') or linear (graded as ' 2 '). Linear fibers were characterized as horizontally arranged fibers. Non-linear fibers appeared haphazardly and in whorls. The density on each of the four sides (distal, proximal, medial, lateral) was graded as loose (grades as ' 1 ') or dense (graded as '2') (Table 1). The senior and junior investigators independently graded the density of fascia in each of the microscope images. Each investigator graded the sides of fascia twice with an hour interval in between investigation. Disagreements between grades on orientation and density of fascia by two investigators were reviewed. The rubric was used to re-grade the density of fascia.

Bland and Altman test was used to determine presence of systematic differences in the three trial linear measurements of the LIS by the senior investigator. The Bland-Altman plot is a graphical method used to compare two measurements techniques. ${ }^{[15]}$ The differences between the two techniques were plotted against the averages of the two techniques. The line of equality was used to determine systematic differences. Lines for $95 \%$ confidence interval (CI) of mean of differences defined the magnitude of systematic differences. If there were significant systematic differences, the line of equality would be outside the $95 \%$ CI of mean of differences.

D'Agostino-Pearson test for normal distribution was used to determine the homogeneity of the mean of the three trial measurements. The mean and $95 \%$ confi-

Table 1

Rubric on characterizing orientation and density of fascia.

\begin{tabular}{lcc}
\hline Items & Dense & Loose \\
\hline Number of bands & $\begin{array}{c}\text { One band of wide } \\
\text { collagen fibers OR } \\
\text { two or more bands } \\
\text { of collagen fibers }\end{array}$ & $\begin{array}{c}\text { One band of narrow } \\
\text { collagen fibers }\end{array}$ \\
\hline Spaces in between bands & $\begin{array}{c}\text { Less space } \\
\text { in between } \\
\text { collagen fibers }\end{array}$ & $\begin{array}{c}\text { More spaces } \\
\text { in between } \\
\text { collagen fibers }\end{array}$ \\
\hline
\end{tabular}




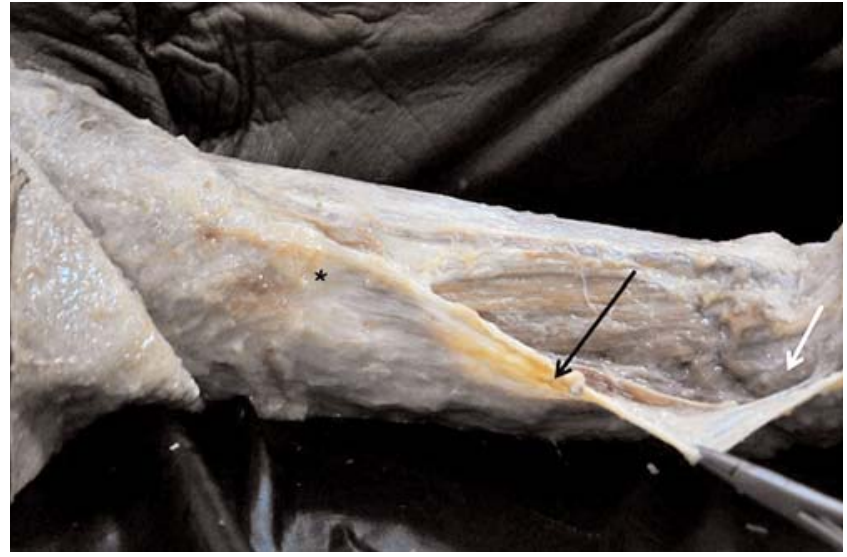

Figure 1. Lateral intermuscular septum (black arrow) from brachioradialis (white arrow) to middle deltoid (*).

dence interval were used to describe the length of LIS. The number of LIS based on attachment sites in the upper arm was noted. Paired samples t-test was used to compare the LIS length between the upper extremities of participants. Based on gender, Independent samples ttest was used to compare length of LIS.

Intra-class correlation coefficients (ICCs) were used to determine the intra-tester and inter-tester reliability of the investigators in grading the density of the fascia. Absolute agreement was used to consider effects of systematic differences in classifying the fascia. Intraclass correlation coefficients (ICCs) were interpreted as follows:

- 0-0.2: poor agreement

- 0.3-0.4: fair agreement

- 0.5-0.6: moderate agreement

- 0.7-0.8: strong agreement

- >0.8: almost perfect agreement

\section{Results}

\section{Linear measurements of LIS}

Using the Bland and Altman plot, no systematic differences and outliers were found in the three trial LIS length measurements by the senior investigator ( $p>0.05$ ). Using the D'Agostino-Pearson test for normal distribution, the three trial measurements were homongenously distributed ( $>>0.05)$. In 17 out of the 19 dissected upper extremities, the LIS attaching from the brachioradialis to middle deltoid (Figure 1) had mean (95\% confidence interval) length measurements of $14.5(13.8-15.1) \mathrm{cm}$. In 2 out of the 19 dissected upper extremities, the LIS attaching from the brachioradialis to the lateral head of

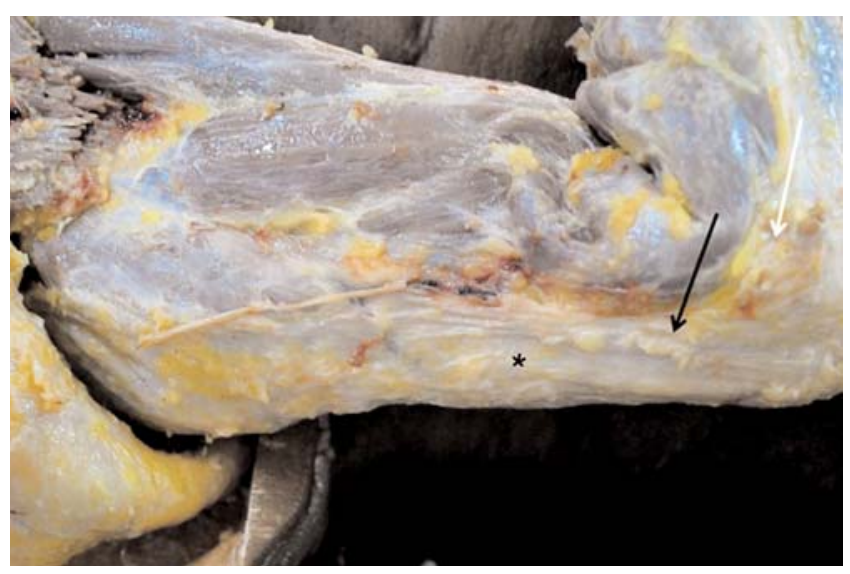

Figure 2. Lateral intermuscular septum (black arrow) from brachioradialis (white arrow) to triceps brachii ${ }^{*}$ ).

the triceps brachii (Figure 2) had mean (95\% confidence interval) length measurements of $10.7(4.7-16.7) \mathrm{cm}$.

Considering that there were sufficient number (17 out of 19) of LIS attaching from the brachioradialis to middle deltoid, the linear measurements were compared based on laterality and gender. Based on laterality and gender, no significant differences in LIS length from brachioradialis to middle deltoid was noted (Table 2).

Figure 3 shows the characteristics of the LIS graded by the senior and junior investigators. Both investigators demonstrated perfect agreement in classifying fibers as linear or not linear $(\mathrm{ICC}=1)$. For intra-tester reliability [ICC $(95 \% \mathrm{CI})]$, the senior and junior investigators demonstrated strong [0.71(0.57-0.81)] and almost perfect agreement [0.92(0.87-0.95)] respectively, in grading the sides of fascia as loose or dense (Figure 3). For inter-tester reliability, both investigators demonstrated moderate agreement in their scores $[\mathrm{ICC}(95 \% \mathrm{CI})=0.50(0.30-0.70)]$. The investigators did not agree in 18/72 grades of fascia density. Disagreements between investigators were on width $(11 / 18)$ and scarcity of fibers (9/18) of collagen bands.

68 out of the 72 fascia images of LIS were non-haphazardly and linearly oriented in relation to the lateral epicondyle. The results on grades on orientation and density of LIS by two investigators were shown in Table 2 . Appendix B lists the images graded by the investigators.

\section{Discussion}

This study describes the length, orientation and density of the lateral intermuscular septum in the upper arm. The cadaver findings on LIS were as follows: a. LIS connected brachioradialis to middle deltoid (17/19 elbows) with mean $(95 \% \mathrm{CI})$ of $14.5(13.8-15.1) \mathrm{cm}$; b. LIS was 

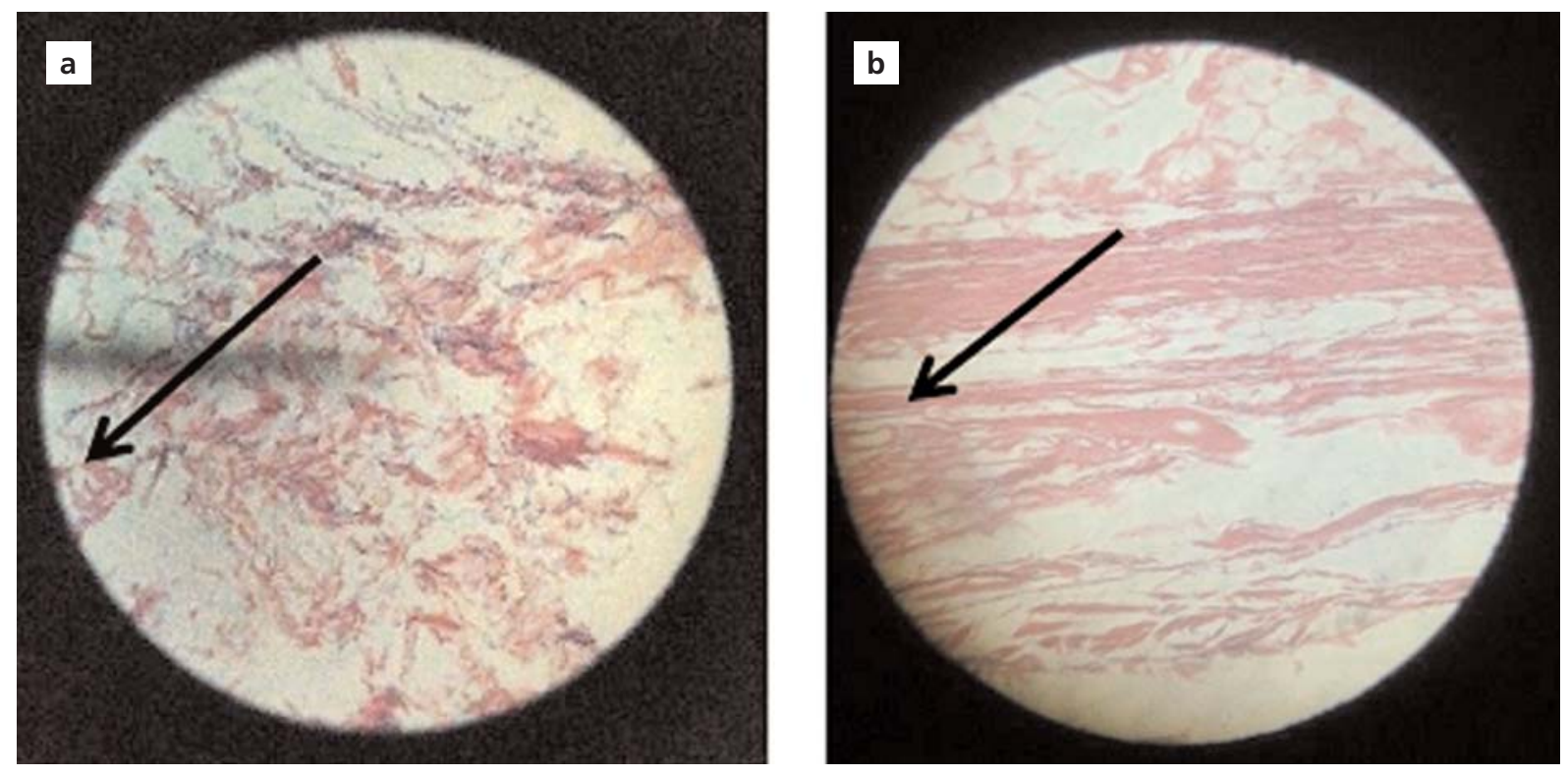

Figure 3. Microscope images of lateral intermuscular septum. (a) Collagen fibers are not-linearly arranged. Arrow points to loose distal side of LIS. (b) Collagen fibers are linearly arranged. Arrow points to the dense distal sides of LIS.

horizontally arranged in the upper arm (68/72 elbows); and c. LIS was convincingly dense on distal (16/18) and loose (12/16) on medial sides of LIS.

The LIS in the upper extremity connected the brachioradialis to the middle deltoid in 17 of 19 upper extremities of cadavers). The LIS connection was reported in the cadaver dissection by Stecco et al. $(2009)^{[16]}$ and Dones et al. ${ }^{[14]}$ However, Dones et al. $(2013)^{[14]}$ only reported this LIS connection in three of the 19 dissected elbows.] Considering that middle deltoid works on the shoulder and the brachioradialis on the elbow, this LIS connection underpins the possible relationship of shoulder and elbow movements. This connection was demonstrated in research by Abbott (2001) where a change in shoulder range of motion following manual therapy to the elbow was noted; there was a significantly restricted shoulder rotary motion at side of LE. ${ }^{[17]}$

The fibers forming the LIS (68/72) were linearly arranged towards the lateral epicondyle. Secondary to the non-haphazard arrangement of the LIS, the traction created by the forearm extensor muscles during handgrip may be effectively communicated to the upper arm through the LIS. The LIS' linear arrangement connecting the brachioradialis and middle deltoid is a microcosm, Myers (2009) reported that the fascia is a fibrous net that connects muscle and bones in the body. ${ }^{[18]}$ Langevin et al. (2005) reported that the body is a web of fascia involved in dynamic, body wide cellular activity reacting towards all externally and internally generated mechanical forces. ${ }^{[19]}$

The dense distal fibers of LIS may underpin the inherent stiffness of the elbow. This density complemented the tight arrangement of the LIS on the lateral aspect of the elbow proposed by Dones et al. (2013). ${ }^{[14]}$ The elbow has a unique role of maintaining stability (by virtue of LIS tightness) while engaged in repetitive and

Table 2

Linear measurements of lateral intermuscular septum in the upper arm.

\begin{tabular}{lccc}
\hline Characteristics & Groups ( $\mathbf{n})$ & Mean $\mathbf{( 9 5 \% ~ C l ) ~ i n ~ c m ~}$ & P-value \\
\hline Laterality & Right (7) & $13.9(12.7-15.3)$ & $>0.05$ \\
& Left (7) & $14.7(13.9-15.3)$ & \\
\hline \multirow{2}{*}{ Gender } & Male (15) & $14.4(13.7-15.1)$ & $>0.05$ \\
& Female (2) & $14.7(9.3-19.9)$ & \\
\hline
\end{tabular}

$\mathrm{Cl}$ : confidence interval.

Table 3

Results on grades on orientation and density of lateral intermuscular septum.

\begin{tabular}{lccc}
\hline Sides of fascia & $\begin{array}{c}\text { Linear/Not Linear } \\
(\mathbf{n}=\mathbf{1 8})\end{array}$ & $\begin{array}{c}\text { Loose } \\
(\mathbf{n}=\mathbf{1 8})\end{array}$ & $\begin{array}{c}\text { Dense } \\
(\mathbf{n}=\mathbf{1 8})\end{array}$ \\
\hline Medial & $17 / 1$ & 12 & 6 \\
Lateral & $17 / 1$ & 9 & 9 \\
Proximal & $17 / 1$ & 9 & 9 \\
Distal & $17 / 1$ & 2 & 16 \\
\hline
\end{tabular}


forceful handgrip tasks. This inherent stiffness in the elbow may be pathologically increased in elbows with LE, associated with lateral elbow pain in LE.

\section{Conclusion}

Considering the dense and non-haphazard LIS arrangement in the upper arm, the LIS contributes to the inherent tightness of the elbow that may be important during handgrip activities. The connection between brachioradialis and middle deltoid by LIS makes it important in considering the changes in shoulder movement associated with lateral elbow pain in LE.

\section{References}

1. Vicenzino B, Cleland JA, Bisset L. Joint manipulation in the management of lateral epicondylalgia: a clinical commentary. J Man Manip Ther 2007;15:50-6.

2. Shiri R, Viikari-Juntura E. Lateral and medial epicondylitis: role of occupation factors. Best practice and research. Best Pract Res Clin Rheumatol 2011;25:43.

3. Lebrun C. What are the best diagnostic criteria for lateral epicondylitis? In: Wright JG, editor. Evidence-based orthopaedics: the best answers to clinical questions. London: Elsevier; 2008. p. $148-57$.

4. Albrecht S, Cordis R, Kleihues H, Noack W. Pathoanatomic findings in radiohumeral epicondylopathy: a combined anatomic and electromyographic study. Arch Orthop Trauma Surg 1997;116:157-63.

5. Roles NC, Maudsley RH. Radial tunnel syndrome: resistant tennis elbow as a nerve entrapment. J Bone Joint Surg Br 1972;54:499508.

6. Van der Wal J. The architecture of the connective tissue in the musculoskeletal system-an often overlooked functional parameter as to proprioception in the locomotor apparatus. Int J Ther Massage Bodywork 2009;2:9-23.
7. Bunata RE, Brown DS, Capelo R. Anatomic factors related to the cause of tennis elbow. J Bone Joint Surg Br 2007;89:1955-63.

8. Oskarsson E, Gustafsson BE, Pettersson K, Aulin KP. Decreased intramuscular blood flow in patients with lateral epicondylitis. Scand J Med Sci Sports 2007;17:211-5.

9. Erak S, Day R, Wang A. The role of supinator in the pathogenesis of chronic lateral elbow pain: a biomechanical study. J Hand Surg Br 2004;29:461-4.

10. Fairbank SM, Corlett RJ. The role of the extensor digitorum communis muscle in lateral epicondylitis. J Hand Surg Br 2002;27: 405-9.

11. De Smet L, Van Raebroeckx T, Van Ransbeeck H. Radial tunnel release and tennis elbow: disappointing results? Acta Orthop Belg 1999;65:510-3.

12. Wilhelm A. Tennis elbow: treatment of resistant cases by denervation. J Hand Surg Br 1996;21:523-33.

13. Muehlberger T, Buschmann A, Ottomann C, Toman N. Aetiology and treatment of a previously denervated "tennis" elbow. Scand J Plast Reconstr Surg Hand Surg 2009;43:50-3.

14. Dones VC III, Milanese S, Worth D, Grimmer-Somers L. The anatomy of the forearm extensor muscles and the fascia in the lateral aspect of the elbow joint complex. Anatomy \& Physiology: Current Research 2013;3:117.

15. Download MedCalc Version 15.2.2. (2015, February 27). Retrieved April 19, 2015 from http://www.medcalc.org/download.php.

16. Stecco C, Porzionato A, Macchi V, Tiengo C, Parenti A, Aldegheri R, Delmas V, De Caro R. A histological study of the deep fascia of the upper limb. Ital J Anat Embryol 2006;111:10510 .

17. Abbott JH. Mobilization with movement applied to the elbow affects shoulder range of movement in subjects with lateral epicondylalgia. Man Ther 2001;6:170-7.

18. Myers TW. Anatomy trains. Myofascial meridians for manual and movement therapists anatomy trains. 2nd ed. New York: Churchill Livingstone; 2009. p. 148-69.

19. Langevin HM, Bouffard NA, Badger GJ, Iatridis JC, Howe AK. Dynamic fibroblast cytoskeletal response to subcutaneous tissue stretch ex vivo and in vivo. Am J Physiol Cell Physiol 2005;288: C747-56.

\begin{tabular}{l} 
Appendix A. \\
Microlab processing of specimen included: \\
\hline - Placement of the specimen in petri-dish with $10 \%$ formalin solution. \\
- Washing of specimen with water until the smell of the solution dissipated. \\
- Placement of the specimen successively in $50 \%$ ethanol, $70 \%$ ethanol, \\
- Washing of the specimen with Xylene solution with addition of paraffin. \\
- Orientation of the paraffin-treated specimen in blocks. \\
- sectioning of the blocked specimens in hot plate known as affixed \\
- Staining and counter-staining of affixed specimen using Hematoxylin \\
- Mound Eosin. \\
- Oven-drying of the specimen on slides. \\
- The affixed specimen was mounted on three separate glass slides.
\end{tabular}



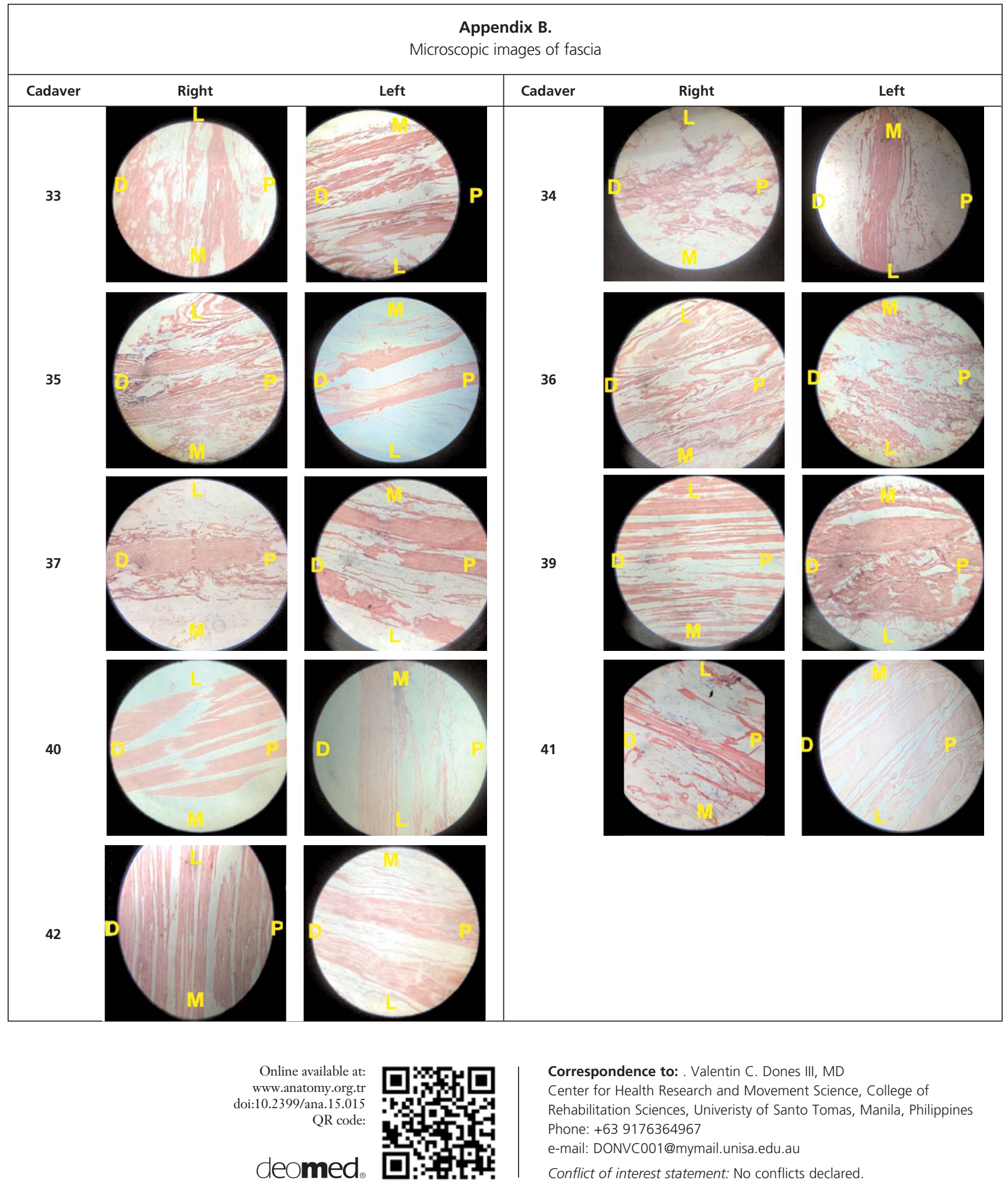

Correspondence to: . Valentin C. Dones III, MD

Center for Health Research and Movement Science, College of

Rehabilitation Sciences, Univeristy of Santo Tomas, Manila, Philippines Phone: +639176364967

e-mail: DONVC001@mymail.unisa.edu.au

Conflict of interest statement: No conflicts declared.

This is an open access article distributed under the terms of the Creative Commons Attribution-NonCommercial-NoDerivs 3.0 Unported (CC BY-NCND3.0) Licence (http://creativecommons.org/licenses/by-nc-nd/3.0/) which permits unrestricted noncommercial use, distribution, and reproduction in any medium, provided the original work is properly cited. Please cite this article as: Dones VC III, Aguinaldo IP, Pulido TMM, Goite LM, Gabrieles MA, Estrella ME, Tan CD, Gargalicana JAD, Limjoco ADC, Sacdalan RJA. Gross anatomical and histological orientation of the lateral intermuscular septum of the upper arm: a descriptive study. Anatomy 2015;9(2):66-71. 